\title{
Fleet maintenance using IOT technology
}

\author{
Surabhi Hatagale ${ }^{1}$, Dr. Ramkrishna Manatkar ${ }^{2}$ \\ ${ }^{1}$ Symbiosis Institute of Operations Management, Nashik, Symbiosis International (Deemed University), Pune, India \\ ${ }^{2}$ Assistant Professor, Symbiosis Institute of Operations Management, Nashik, Symbiosis International (Deemed University), \\ Pune, India
}

\begin{abstract}
In fleet management, fleet maintenance is an important exposure to increase availability. Periodic and preventive maintenance is one such crucial aspect which is considered regardless of the practical faults which in sets of the need for repair and replacement cost as well time attached with it. With development in technology IOT and big data have been in talks. With all the data that is being produced predictive maintenance can be performed using this technology. IOT based predictive maintenance can increase fleet availability, stability and efficiency, reduced cost through effective maintenance planning and eliminate unnecessary maintenance tasks.
\end{abstract}

Keywords :

Fleet maintenance, Fleet management, telematics, predictive maintenance

Article Received: 10 August 2020, Revised: 25 October 2020, Accepted: 18 November 2020

\section{Introduction}

E-commerce has seen amazing growth in recent Supply chain management is a process for satisfying customer needs and reducing the cost of supply chain across the activities of suppliers and consumers, right from the procurement of raw materials to product manufacturing, transport and shipment, and sales. Reduced transport cost which includes the fleet maintenance and management accounts to $1 / 3^{\text {rd }}$ of the operating cost. Thus fleet management is crucial for cost reduction which helps in improvising fleet scheduling, operational efficiency and effectiveness. Which also includes information management for fleet maintenance. The activities included in fleet maintenance management include allocating and scheduling of resources based on repair, replacement and preventive maintenance. Fleet operators and companies who own huge fleet spend a large amount of money, time and resources in effectively managing the fleet assets to ensure safety and desired performance levels.

\section{Literature review}

IOT (Internet of Things) is a new prototype which has emerged rapidly connecting billions of devices through internet. IOT applications has helped us to build smart farming, smart transport, smart health, smart cities, smart homes and smart grids, where are the devices in particular systems are connected through internet and big data becomes prevalent.

IOT can be revolutionized in fleet management in various ways where we can carry out environment friendly operations which helps to monitor the driver performance and monitor fuel utilization along with all standard features of fleet management. Over speeding the vehicle or keeping them idle have an harmful effect on the environment, apart from that they also cause wear and tear and additional fuel cost. Thus using IOT technology, we can easily track driver's behavior and get real time automated alerts. Sensor equipped vehicles sending automated signals and early warning alerts for low battery, coolant temperature, engine maintenance can help in providing preventive maintenance which helps in longer health of a vehicle.

Vehicle performance has a very pivotal impact on fleet efficiency. IOT helps the engine remotely monitor and analyze critical engine parameters, diagnostic parameters and integrate it with field engineer optimization and spare parts management. Various processes are automated using IOT in fleet management such as the system can send ticket notification to tow trucks, nearest service stations, gather data about the route, traffic, vehicle speed and generate alerts and display messages to help the passengers to plan their journey accordingly. 
Traffic maintenance can be improved used RFID technology and Raspberry pi where raspberry pi is mounted in the vehicle and is interfaced with the sensors like gas sensors, temperature sensor and stun sensors. These sensors are mounted for beforehand mishaps. When the mishap happens the value of the sensor changes and a message is sent to the predefined number or emergency vehicle with help of GSM.

As the message is received by the emergency vehicle, a reasonable course is given. The vehicle includes controller ARM that is interfaced with RFID which send electromagnetic waves. As the car arrives at the light RFID perusers locates the electromagnetic influxes of the tag. During traffic sign red, the perusers experiences the database in portion of seconds and switch the red light green. This helps in an unmistakable course in emergency vehicle. GSM and microcontrollers are the systems that help to organize motors of vehicles.

There is proposed framework for the management of transportation resource management involved to provide efficient fleet management based on the user's requirements.

The overall framework includes three layers for resource management and predictive maintenance.

\section{Fleet to IOT layer}

This is the first layer of framework which has necessary information of the vehicle and the GPS configuration which represents the IOT information for fleet management. It abstracts the fog and the embedded systems. It performs sensing, light weight storage, networking.

\section{Fleet to Web layer}

This layer deals with user interface and cloud server configuration. The user interface communication are managed in this layer. The IOT configuration and the user accepts the input and output management of the vehicle and thus performs more heavy duty storage and machine learning.

\section{Fleet for data maintenance layer}

This layer includes vehicle information maintenance for automatic updation of necessary information. Various factors of information are included in this layer such as engine temperature, oil level, fuel observation/consumption, location etc. based on which the information of fleet data is sustained in cloud server.

Key objectives include

a. Fuel level is monitored

b. Measurement of fuel utilization

c. Increase the vehicle performance

In order to maintain vehicle health engine diagnostic with IOT is used to overcome traditional engine diagnostic systems. It is a system that sends information over Onboard diagnosis connection employing wireless system that connects to the web. Using this data we can measure the above mentioned criteria of the engine.

\section{Detection of driver's help}

IOT helps to provide information of the behavior of the driver with help of live camera monitoring. It helps to monitor behavioral information with an aspect to check unnecessary activities of the driver or theft activities. With this there is a possibility of resource wastage and alertness in the behavior of the driver.

\section{Tracking the location of the vehicle}

This section helps to provide the live location of the vehicle with map representation with highest accuracy. Data from various sensors are sent to the local gateway as the vehicle moves. With the help of IOT and GPS tracking system where the fleet managers have the information of the weather conditions, road conditions and traffic.

Predictive Fleet maintenance

Artificial intelligence plays a vital role in building predictions based on collected telematics data.

Using telematics data, the fleet companies can shift their service from reractive to preventive measures which can track the mechanical failures in real time and improve performace efficiency 


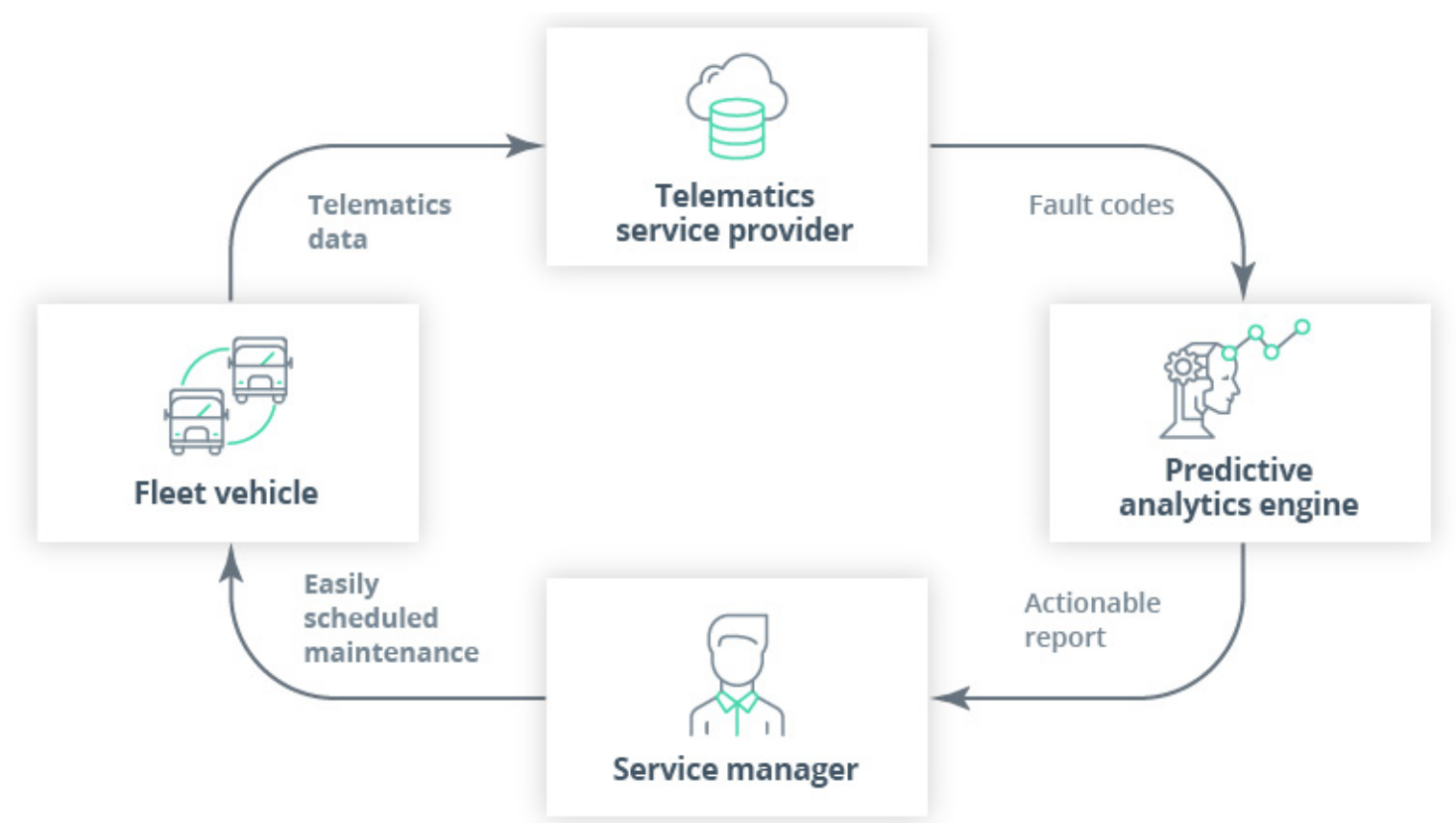

\section{Conclusion}

Thus the IOT in fleet management works as an automation for fleet management as it helps to reduce downtime and increase vehicle availability. It has also increased the fleet efficiency where there is continuous monitoring of each vehicle resulting in individual vehicle profile. It will also reduce fuel consumption as proper maintenance saves fuel. It basically eases out process of maintenance and increases fleet life.

\section{References}

[1] IoT-based predictive maintenance for fleet management Patrick Killeena,_, Bo Dingb, Iluju Kiringaa, Tet Yeap

[2] Fleet Automation using IoT Logistics R.Mahalakshmi Priya, M.Vasumathi, K.Sathish Kumar, M.Arun, S.Pandikumar

[3] https://www.clariontech.com/blog/iot-isrevolutionizing-fleet-management-here-ishow https://www.ijeat.org/wpcontent/uploads/papers/v8i6/F8911088619.p df

[4] L. C. M. Varandas, B. Vaidya, and J. J. P. C. Rodrigues, "mTracker : A Mobile Tracking Application for Pervasive Environment," in 24th International Conference on Advanced Information Networking and Applications Workshops, 2010, pp. 962-967.
[5] https://www.pratititech.com/blog/fleetmanagement-and-iot-unlimited-potentialfor-smart-transformation

[6] https://blog.fleetcomplete.com/role-of-iotfleet-management https://fleetroot.com/blog/how-does-iotassist-in-fleet-management/

[7] https://www.intellias.com/how-to-apply-ai-forpredictive-fleet-management-maintenance-software/

[8] ] Amarasinghe, M., Kottegoda, S., Arachchi, A.L., Muramudalige, S., Bandara, H.M.N.D., Azeez, A., 2015. Cloud-based driver monitoring and vehicle diagnostic with obd2 telematics. 2015 Fifteenth International Conference on Advances in ICT for Emerging Regions (ICTer) doi:10.1109/icter.2015.7377695

[9] Byttner, S., Nowaczyk, S., Prytz, R., Rognvaldsson, T., 2013. A field test with self-organized modeling for knowledge discovery in a fleet of city buses. 2013 IEEE International Conference on Mechatronics and Automation doi:10.1109/icma.2013.6618034.

[10] Ding, B., Mends, D., Kiringa, I., Yeap, T., . Iot architecture with hybrid communication modes for fleet management, in: Technical report, Faculty of Engineering, University of Ottawa, Ottawa, Ontario, Canada 